\title{
Measuring Performance of Ratio-Exponential-Log Type General Class of Estimators Using Two Auxiliary Variables
}

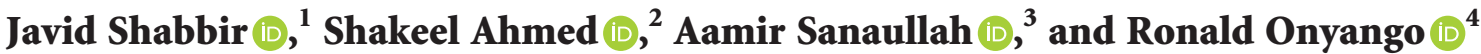 \\ ${ }^{1}$ Department of Statistics, Quaid-i-Azam University, Islamabad 45320, Pakistan \\ ${ }^{2}$ School of Natural Sciences, National University of Sciences and Technology (NUST), Islamabad, Pakistan \\ ${ }^{3}$ Department of Statistics, COMSATS University Islamabad, Lahore Campus, Islamabad, Pakistan \\ ${ }^{4}$ Department of Applied Statistics, Financial Mathematics and Actuarial Science, \\ Jaramogi Oginga Odinga University of Science and Technology, Bondo, Kenya
}

Correspondence should be addressed to Ronald Onyango; assangaronald@gmail.com

Received 27 July 2021; Accepted 17 September 2021; Published 1 October 2021

Academic Editor: Ishfaq Ahmad

Copyright (C) 2021 Javid Shabbir et al. This is an open access article distributed under the Creative Commons Attribution License, which permits unrestricted use, distribution, and reproduction in any medium, provided the original work is properly cited.

In this paper, a ratio-exponential-log type general class of estimators is proposed in estimating the finite population mean using two auxiliary variables when population parameters of the auxiliary variables are known. From the proposed estimator, some special estimators are identified as members of the proposed general class of estimators. The mean square error (MSE) expressions are obtained up to the first order of approximation. This study finds that the proposed general class of estimators outperforms as compared to the conventional mean estimator, usual ratio estimators, exponential-ratio estimators, log-ratio type estimators, and many other competitor regression type estimators. Four real-life applications are used for efficiency comparison.

\section{Introduction}

In survey sampling, ratio, product, exponential-ratio, logratio, and regression type estimators are modified or constructed by many researchers to enhance the precision of the estimators under different sampling designs by using the auxiliary variables. These estimators are commonly used by taking the advantage of correlation coefficient between the study variable and the auxiliary variable(s). Some notable work by the authors includes Olkin [1]; Mohanty [2]; AbuDayyeh et al. [3]; Koyuncu and Kadilar [4]; Swain [5]; Lu and Yan [6]; Lu et al. [7]; Sanaullah et al. [8]; Lu [9]; Muneer et al. [10]; Shabbir and Gupta [11]; Akingbade and Okafor [12]; Shabbir et al. [13]; Shabbir et al. [14]; Bhushan et al. [15]; Lone et al. [16]; and Kumari and Thaur [17].

Consider a finite population $\Gamma=\left\{\Gamma_{1}, \Gamma_{2}, \ldots, \Gamma_{N}\right\}$ of $N$ units. A sample of size $n$ units is drawn from a population by using simple random sampling without replacement (SRSWOR). Let $y_{i}$ and $\left(x_{i}, z_{i}\right)$ be the characteristics of the study variable $(Y)$ and the auxiliary variables $(X, Z)$, respectively. Let $\bar{y}=n^{-1} \sum_{i=1}^{n} y_{i}$, and $\left(\bar{x}=n^{-1} \sum_{i=1}^{n} x_{i}, \bar{z}=\right.$ $\left.n^{-1} \sum_{i=1}^{n} z_{i}\right)$, respectively, be the sample means corresponding to the population means $\bar{Y}=N^{-1} \sum_{i=1}^{N} y_{i}$, and $\left(\bar{X}=N^{-1} \sum_{i=1}^{N} x_{i}, \bar{Z}=N^{-1} \sum_{i=1}^{N} z_{i}\right)$. To obtain the bias and MSE expressions, we define the following error terms: $\Xi_{0}=(\bar{y} / \bar{Y})-1$, and $\Xi_{1}=(\bar{x} / \bar{X})-1, \Xi_{2}=(\bar{z} / \bar{Z})-1$, such that $E\left(\Xi_{i}\right)=0,(i=0,1,2), E\left(\Xi_{0}^{2}\right)=\Theta C_{y}^{2}, \quad E\left(\Xi_{1}^{2}\right)=\Theta C_{x}^{2}$, $E\left(\Xi_{2}^{2}\right)=\Theta C_{z}^{2}, \quad E\left(\Xi_{0} \Xi_{1}\right)=\Theta C_{y x}, \quad E\left(\Xi_{0} \Xi_{2}\right)=\Theta C_{y z}$, and $E\left(\Xi_{1} \Xi_{2}\right)=\Theta C_{x z}$ where $\Theta=\left(n^{-1}-N^{-1}\right), \quad C_{y}=\bar{Y}^{-1} S_{y}$, $C_{x}=\bar{X}^{-1} S_{x}, C_{z}=\bar{Z}^{-1} S_{z}, C_{y x}=\rho_{y x} C_{y} C_{x}, C_{y z}=\rho_{y z} C_{y} C_{z}$, $C_{x z}=\rho_{x z} C_{x} C_{z}, \quad \rho_{y x}=\left(S_{y} S_{x}\right)^{-1} S_{y x}, \quad \rho_{y z}=\left(S_{y} S_{z}\right)^{-1} S_{y z}$, $\rho_{x z}=\left(S_{x} S_{z}\right)^{-1} S_{x z} S_{y}=\sqrt{(N-1)^{-1} \sum_{i=1}^{N}\left(y_{i}-\bar{Y}\right)^{2}}, \quad S_{x}=$ $\sqrt{(N-1)^{-1} \sum_{i=1}^{N}\left(x_{i}-\bar{X}\right)^{2}}, S_{z}=\sqrt{(N-1)^{-1} \sum_{i=1}^{N}\left(z_{i}-\bar{Z}\right)^{2}}$, $S_{y x}=(N-1)^{-1} \sum_{i=1}^{N}\left(y_{i}-\bar{Y}\right)\left(x_{i}-\bar{X}\right), \quad S_{y z}=(N-1)^{-1}$ $\sum_{i=1}^{N}\left(y_{i}-\bar{Y}\right)\left(z_{i}-\bar{Z}\right)$, and $S_{x z}=(N-1)^{-1} \sum_{i=1}^{N}\left(x_{i}-\bar{X}\right)$ $\left(z_{i}-\bar{Z}\right)$. 


\section{Some Existing Estimators}

Some existing estimators available in the literature are essential to be discussed here.

2.1. Sample Mean Estimator. The usual sample mean estimator and its variance are given as

$$
\begin{gathered}
\hat{\bar{Y}}_{(0)}=\bar{y}, \\
\operatorname{Var}\left(\widehat{\bar{Y}}_{(0)}\right)=\Theta \bar{Y}^{2} C_{y}^{2} .
\end{gathered}
$$

2.2. Ratio Estimators. The usual ratio estimators when using single and two auxiliary variables are given by

$$
\begin{aligned}
& \text { (i) } \hat{\bar{Y}}_{(R)}^{(1)}=\bar{y}\left(\frac{\bar{X}}{\bar{x}}\right), \\
& \text { (ii) } \hat{\bar{Y}}_{(R)}^{(2)}=\bar{y}\left(\frac{\bar{Z}}{\bar{x}}\right), \\
& \text { (iii) } \hat{\bar{Y}}_{(R)}^{(3)}=\bar{y}\left(\frac{\bar{X}}{\bar{x}}\right)\left(\frac{\bar{Z}}{\bar{z}}\right) .
\end{aligned}
$$

The MSEs of ratio estimators $\widehat{\bar{Y}}_{(R)}^{(i)}(i=1,2,3)$ to first order of approximation are given by

$$
\begin{aligned}
& \text { (i) } \operatorname{MSE}\left(\hat{\bar{Y}}_{(R)}^{(1)}\right) \cong \Theta \bar{Y}^{2}\left[C_{y}^{2}+C_{x}^{2}-2 C_{y x}\right] \\
& \text { (ii) } \operatorname{MSE}\left(\hat{\bar{Y}}_{(R)}^{(2)}\right) \cong \Theta \bar{Y}^{2}\left[C_{y}^{2}+C_{z}^{2}-2 C_{y z}\right] \\
& \text { (iii) } \operatorname{MSE}\left(\hat{\bar{Y}}_{(R)}^{(3)}\right) \cong \Theta \bar{Y}^{2}\left[C_{y}^{2}+C_{x}^{2}+C_{z}^{2}-2\left(C_{y x}+C_{y z}\right)+2 C_{x z}\right]
\end{aligned}
$$

The ratio estimators $\widehat{\bar{Y}}_{(R)}^{(i)}(i=1,2,3)$ are performing better than $\bar{Y}_{(0)}$ under certain conditions.

2.3. Exponential-Ratio Estimators. The usual exponentialratio estimators when using single and two auxiliary variables are given by

$$
\begin{aligned}
& \text { (i) } \hat{\bar{Y}}_{(E)}^{(1)}=\bar{y} \exp \left(\frac{\bar{X}-\bar{x}}{\bar{X}+\bar{x}}\right), \\
& \text { (ii) } \hat{\bar{Y}}_{(E)}^{(2)}=\bar{y} \exp \left(\frac{\bar{Z}-\bar{z}}{\bar{Z}+\bar{z}}\right), \\
& \text { (iii) } \hat{\bar{Y}}_{(E)}^{(3)}=\bar{y} \exp \left(\frac{\bar{X}-\bar{x}}{\bar{X}+\bar{x}}\right) \exp \left(\frac{\bar{Z}-\bar{z}}{\bar{Z}+\bar{z}}\right) .
\end{aligned}
$$

The MSEs of exponential-ratio estimators $\hat{\bar{Y}}_{(E)}^{(i)}$ $(i=1,2,3)$ to first order of approximation are given by (i) $\operatorname{MSE}\left(\hat{\bar{Y}}_{(E)}^{(1)}\right) \cong \Theta \bar{Y}^{2}\left[C_{y}^{2}+\frac{1}{4} C_{x}^{2}-C_{y x}\right]$,

(ii) $\operatorname{MSE}\left(\widehat{\bar{Y}}_{(E)}^{(2)}\right) \cong \Theta \bar{Y}^{2}\left[C_{y}^{2}+\frac{1}{4} C_{z}^{2}-C_{y z}\right]$,

(iii) $\operatorname{MSE}\left(\widehat{\bar{Y}}_{(E)}^{(3)}\right) \cong \Theta \bar{Y}^{2}\left[C_{y}^{2}+\frac{1}{4}\left(C_{x}^{2}+C_{z}^{2}\right)-\left(C_{y x}+C_{y z}\right)+\frac{1}{2} C_{x z}\right]$.

The exponential-ratio estimators $\hat{\bar{Y}}_{(E)}^{(i)}(i=1,2,3)$ are performing better than $\hat{\bar{Y}}_{(0)}$ and $\hat{\bar{Y}}_{(R)}^{(i)} \quad(i=1,2,3)$ under certain conditions.

2.4. Log-Ratio Estimators. Recently many log-type estimators have appeared in the literature in various forms when the logarithmic relationship between the study variable and the auxiliary variables exists.

The usual log-ratio estimators when using single and two auxiliary variables are given by

$$
\begin{aligned}
& \text { (i) } \hat{\bar{Y}}_{(\log )}^{(1)}=\bar{y}\left[1+\log \left(\frac{\bar{X}}{\bar{x}}\right)\right], \\
& \text { (ii) } \hat{\bar{Y}}_{(\log )}^{(2)}=\bar{y}\left[1+\log \left(\frac{\bar{Z}}{\bar{z}}\right)\right], \\
& \text { (iii) } \hat{\bar{Y}}_{(\log )}^{(3)}=\bar{y}\left[1+\log \left(\frac{\bar{X}}{\bar{x}}\right)\right]\left[1+\log \left(\frac{\bar{Z}}{\bar{z}}\right)\right] .
\end{aligned}
$$

The MSEs of log-ratio estimators $\hat{\bar{Y}}_{(\log )}^{(i)}(i=1,2,3)$ to first order of approximation are given by

(i) $\operatorname{MSE}\left(\hat{\bar{Y}}_{(\log )}^{(1)}\right) \cong \Theta \bar{Y}^{2}\left[C_{y}^{2}+C_{x}^{2}-2 C_{y x}\right]$,

(ii) $\operatorname{MSE}\left(\hat{\bar{Y}}_{(\log )}^{(2)}\right) \cong \Theta \bar{Y}^{2}\left[C_{y}^{2}+C_{z}^{2}-2 C_{y z}\right]$,

(iii) $\operatorname{MSE}\left(\widehat{\bar{Y}}_{(\log )}^{(3)}\right) \cong \Theta \bar{Y}^{2}\left[C_{y}^{2}+C_{x}^{2}+C_{z}^{2}-2\left(C_{y x}+C_{y x}\right)+2 C_{x z}\right]$.

The MSEs of log-ratio estimators $\widehat{\bar{Y}}_{(\log )}^{(i)}(i=1,2,3)$ are exactly equal to the MSEs of ratio estimators $\widehat{\bar{Y}}_{(R)}^{(i)}(i=1,2,3)$ but their biases are different (not shown here).

2.5. Regression Estimators. The usual regression estimators when using single and two auxiliary variables are given by

$$
\begin{aligned}
& \text { (i) } \hat{\bar{Y}}_{(\mathrm{Reg})}^{(1)}=\bar{y}+b_{y x}(\bar{X}-\bar{x}), \\
& \text { (ii) } \hat{\bar{Y}}_{(\mathrm{Reg})}^{(2)}=\bar{y}+b_{y z}(\bar{Z}-\bar{z}), \\
& \text { (iii) } \hat{\bar{Y}}_{(\mathrm{Reg})}^{(3)}=\bar{y}+b_{y x}(\bar{X}-\bar{x})+b_{y z}(\bar{Z}-\bar{z}),
\end{aligned}
$$

where $b_{y x}=\left(s_{y x} / s_{x}^{2}\right)$ and $b_{y z}=\left(s_{y z} / s_{z}^{2}\right)$ are the sample regression coefficients. 
The MSEs of regression estimators $\hat{\bar{Y}}_{(\mathrm{Reg})}^{(i)}(i=1,2,3)$ are given by

(i) $\operatorname{MSE}\left(\widehat{\bar{Y}}_{(\mathrm{Reg})}^{(1)}\right) \cong \Theta \bar{Y}^{2} C_{y}^{2}\left(1-\rho_{y x}^{2}\right)$,

(ii) $\operatorname{MSE}\left(\hat{\bar{Y}}_{(\mathrm{Reg})}^{(2)}\right) \cong \Theta \bar{Y}^{2} C_{y}^{2}\left(1-\rho_{y z}^{2}\right)$,

(iii) $\operatorname{MSE}\left(\hat{\bar{Y}}_{(\mathrm{Reg})}^{(3)}\right) \cong \Theta \bar{Y}^{2} C_{y}^{2}\left(1-\rho_{y x}^{2}-\rho_{y z}^{2}+2 \rho_{y x} \rho_{y z} \rho_{x z}\right)$.

These regression estimators $\widehat{\bar{Y}}_{(\mathrm{Reg})}^{(i)}(i=1,2,3)$ are performing better than $\widehat{\bar{Y}}_{(0)}$ and $\widehat{\bar{Y}}_{(j)}^{(i)} \quad(i=1,2,3 ; j=R, E$, $\log )$ under certain conditions.

2.6. Some More Regression Type Estimators. Mohanty [2] suggested the following regression-type estimator:

$$
\hat{\bar{Y}}_{(\operatorname{Reg})}^{(M)}=\bar{y}+b_{y x}(\bar{X}-\bar{x})\left(\frac{\bar{Z}}{\bar{z}}\right) \text {. }
$$

The MSE of $\hat{\bar{Y}}_{(\mathrm{Reg})}^{(M)}$ is given by

$\operatorname{MSE}\left(\hat{\bar{Y}}_{(\mathrm{Reg})}^{(M)}\right) \cong \Theta \bar{Y}^{2}\left[C_{y}^{2}\left(1-\rho_{y x}^{2}\right)+C_{z}^{2}-2 C_{y z}+2 C_{y} C_{z} \rho_{y x} \rho_{x z}\right]$.

Swain [5] introduced the following regression-type estimator

$$
\widehat{\bar{Y}}_{(\operatorname{Reg})}^{(S)}=\left[\bar{y}+d_{0}(\bar{X}-\bar{x})\right]\left(\frac{\bar{Z}}{\bar{z}}\right)
$$

where $d_{0}$ is constant.

The minimum MSE of $\hat{\bar{Y}}_{(\mathrm{Reg})}^{(S)}$ at optimum value of $d_{0 \text { (opt) }}=\left(\bar{Y}\left(C_{y} \rho_{y x}-C_{z} \rho_{x z}\right) / \bar{X} C_{x}\right)$ is given by

$$
\operatorname{MSE}\left(\hat{\bar{Y}}_{(\mathrm{Reg})}^{(S)}\right)_{\min } \cong \Theta \bar{Y}^{2} C_{y}^{2}\left[\left(C_{y}^{2}+C_{z}^{2}-2 C_{y z}\right)-\left(C_{y} \rho_{y x}-C_{z} \rho_{x z}\right)^{2}\right] \text {. }
$$

The unbiased regression estimator when using two auxiliary variables is given by

$$
\hat{\bar{Y}}_{(\mathrm{Reg})}^{(U)}=\bar{y}+d_{1}(\bar{X}-\bar{x})+d_{2}(\bar{Z}-\bar{z}),
$$

where $d_{1}$ and $d_{2}$ are constants.

The minimum MSE of $\widehat{\bar{Y}}_{(\mathrm{Reg})}^{(U)}$ at optimum values of $d_{1 \text { (opt) }}=\left(S_{y}\left(\rho_{y x}-\rho_{y z} \rho_{x z}\right) / S_{x}\left(1-\rho_{x z}^{2}\right)\right)$ and $d_{2(\mathrm{opt})}=$ $\left(S_{y}\left(\rho_{y z}-\rho_{y x} \rho_{x z}\right) / S_{z}\left(1-\rho_{x z}^{2}\right)\right)$ is given by

$$
\operatorname{MSE}\left(\hat{\bar{Y}}_{(\mathrm{Reg})}^{(U)}\right)_{\min } \cong \Theta \bar{Y}^{2} C_{y}^{2}\left(1-R_{y \cdot x z}^{2}\right) \text {, }
$$

where $R_{y \cdot x z}^{2}=\left(\left(\rho_{y x}^{2}+\rho_{y z}^{2}-2 \rho_{y x} \rho_{y z} \rho_{x z}\right) /\left(1-\rho_{x z}^{2}\right)\right)$ is the multiple correlation coefficient.

\section{Proposed General Class of Estimators}

We propose a ratio-exponential-log type general class of estimators in estimating the finite population mean using two auxiliary variables when some parameters of the auxiliary variables are known. We also obtain different special estimators as members of the general class of estimators which are useful in different real-life situations. The proposed estimator is the combination of three special estimators including ratio, exponential-ratio, and log-ratio by using the linear transformation as

$$
\widehat{\bar{Y}}_{(M)}^{\left(\alpha_{1}, \alpha_{2}, \alpha_{3}, \gamma_{1}, \gamma_{2}, \gamma_{3}\right)}=\bar{y}\left[M_{1}\left\{\frac{\bar{X}^{*}}{\bar{x}^{*}}\right\}^{\alpha_{1}}\left\{\exp \left(\frac{\bar{X}^{*}-\bar{x}^{*}}{\bar{X}^{*}+\bar{x}^{*}}\right)\right\}^{\alpha_{2}}\left\{1+\log \left(\frac{\bar{X}^{*}}{\bar{x}^{*}}\right)\right\}^{\alpha_{3}}+M_{2}\left\{\frac{\bar{Z}^{*}}{\bar{z}^{*}}\right\}^{\gamma_{1}}\left\{\exp \left(\frac{\bar{Z}^{*}-\bar{z}^{*}}{\bar{Z}^{*}+\bar{z}^{*}}\right)\right\}^{\gamma_{2}}\left\{1+\log \left(\frac{\bar{Z}^{*}}{\bar{z}^{*}}\right)\right\}^{\gamma_{3}}\right]
$$

where $M_{i}(i=1,2)$ are constants, whose values are to be determined; $\alpha_{i}(i=1,2,3)$ and $\gamma_{i}(i=1,2,3)$ are scaler quantities; and $\bar{x}^{*}=a \bar{x}+b, \bar{X}^{*}=a \bar{X}+b, \bar{z}^{*}=c \bar{z}+d$, and $\bar{Z}^{*}=c \bar{Z}+d$. Here $a, b, c, d$ are the known population parameters of the auxiliary variables which may be coefficients of variation $\left(C_{x}, C_{z}\right)$, coefficients of kurtosis $\left(\beta_{2 x}, \beta_{2 z}\right)$ and correlation coefficients $\left(\rho_{y x}, \rho_{y z}\right)$.

Solving (25) in terms of errors to the first order of approximation, we have

$$
\widehat{\bar{Y}}_{(M)}^{\left(\alpha_{1}, \alpha_{2}, \alpha_{3}, \gamma_{1}, \gamma_{2}, \gamma_{3}\right)}-\bar{Y} \cong \bar{Y}\left[M_{1}\left\{1+\Xi_{0}-\Delta_{1} g_{1} \Xi_{1}-\Delta_{1} g_{1} \Xi_{0} \Xi_{1}+\Delta_{2} g_{1}^{2} \Xi_{1}^{2}\right\}+M_{2}\left\{1+\Xi_{0}-\Omega_{1} g_{2} \Xi_{2}-\Omega_{1} g_{2} \Xi_{0} \Xi_{2}+\Delta_{2} g_{2}^{2} \Xi_{2}^{2}\right\}-1\right],
$$

where 


$$
\begin{aligned}
& g_{1}=\frac{a \bar{X}+b}{a \bar{x}+b}, \\
& g_{2}=\frac{c \bar{Z}+d}{c \bar{z}+d}, \\
& \Delta_{1}=\alpha_{1}+\frac{1}{2} \alpha_{2}+\alpha_{3}, \\
& \Delta_{2}=\alpha_{1} \alpha_{3}+\frac{1}{2} \alpha_{2} \alpha_{3}+\frac{1}{2} \alpha_{1}\left(\alpha_{1}+1\right)+\frac{1}{8} \alpha_{2}\left(\alpha_{2}+2\right)+\frac{1}{2} \alpha_{1} \alpha_{2}+\frac{1}{2} \alpha_{3}^{2}, \\
& \Omega_{1}=\gamma_{1}+\frac{1}{2} \gamma_{2}+\gamma_{3}, \\
& \Omega_{2}=\gamma_{1} \gamma_{3}+\frac{1}{2} \gamma_{2} \gamma_{3}+\frac{1}{2} \gamma_{1}\left(\gamma_{1}+1\right)+\frac{1}{8} \gamma_{2}\left(\gamma_{2}+2\right)+\frac{1}{2} \gamma_{1} \gamma_{2}+\frac{1}{2} \gamma_{3}^{2} .
\end{aligned}
$$

The bias of $\hat{\bar{Y}}_{(M)}^{\left(\alpha_{1}, \alpha_{2}, \alpha_{3}, \gamma_{1}, \gamma_{2}, \gamma_{3}\right)}$ to the first order of approximation is given by

$$
\begin{aligned}
\operatorname{Bias}\left(\widehat{\bar{Y}}_{(M)}^{\left(\alpha_{1}, \alpha_{2}, \alpha_{3}, \gamma_{1}, \gamma_{2}, \gamma_{3}\right)}\right) \cong & \bar{Y}\left[M_{1}\left\{1+\Theta\left(\Delta_{2} g_{1}^{2} C_{x}^{2}-\Delta_{1} g_{1} C_{y x}\right)\right\}\right. \\
& \left.+M_{2}\left\{1+\Theta\left(\Omega_{2} g_{2}^{2} C_{z}^{2}-\Omega_{1} g_{2} C_{y z}\right)\right\}-1\right] .
\end{aligned}
$$

The MSE of $\hat{\bar{Y}}_{(M)}^{\left(\alpha_{1}, \alpha_{2}, \alpha_{3}, \gamma_{1}, \gamma_{2}, \gamma_{3}\right)}$ to the first order of approximation is given by

$$
\begin{aligned}
\operatorname{MSE}\left(\hat{\bar{Y}}_{(M)}^{\left(\alpha_{1}, \alpha_{2}, \alpha_{3}, \gamma_{1}, \gamma_{2}, \gamma_{3}\right)}\right) \cong & \bar{Y}^{2} E\left[M_{1}\left\{1+\Xi_{0}-\Delta_{1} g_{1} \Xi_{1}-\Delta_{1} g_{1} \Xi_{0} \Xi_{1}+\Delta_{2} g_{1}^{2} \Xi_{1}^{2}\right\}\right. \\
& \left.+M_{2}\left\{1+\Xi_{0}-\Omega_{1} g_{2} \Xi_{2}-\Omega_{1} g_{2} \Xi_{0} \Xi_{2}+\Delta_{2} g_{2}^{2} \Xi_{2}^{2}\right\}-1\right]^{2}
\end{aligned}
$$

Solving (29), we get

$$
\operatorname{MSE}\left(\widehat{\bar{Y}}_{(M)}^{\left(\alpha_{1}, \alpha_{2}, \alpha_{3}, \gamma_{1}, \gamma_{2}, \gamma_{3}\right)}\right) \cong \bar{Y}^{2}\left[1+M_{1}^{2} A_{m}+M_{2}^{2} B_{m}-2 M_{1} C_{m}-2 M_{2} D_{m}+2 M_{1} M_{2} E_{m}\right]
$$

where

$$
\begin{aligned}
& A_{m}=1+\Theta\left\{C_{y}^{2}\left(\Delta_{1}^{2}+2 \Delta_{2}\right) g_{1}^{2} C_{x}^{2}-4 \Delta_{1} g_{1} C_{y x}\right\}, \\
& B_{m}=1+\Theta\left\{C_{y}^{2}\left(\Omega_{1}^{2}+2 \Omega_{2}\right) g_{2}^{2} C_{z}^{2}-4 \Omega_{1} g_{2} C_{y z}\right\}, \\
& C_{m}=1+\Theta\left\{\Delta_{2} g_{1}^{2} C_{x}^{2}-\Delta_{1} g_{1} C_{y x}\right\} \\
& D_{m}=1+\Theta\left\{\Omega_{2} g_{2}^{2} C_{z}^{2}-\Omega_{1} g_{2} C_{y z}\right\}, \\
& E_{m}=1+\Theta\left\{C_{y}^{2}+\Delta_{2} g_{1}^{2} C_{x}^{2}+\Omega_{2} g_{2}^{2} C_{z}^{2}-2 \Delta_{1} g_{1} C_{y x}-2 \Omega_{1} g_{2} C_{y z}+\Delta_{1} \Omega_{1} g_{1} g_{2} C_{x z}\right\}
\end{aligned}
$$


Solving (30), the optimum values $M_{i}(i=1,2)$ are given as

$$
\begin{aligned}
& M_{1(\mathrm{opt})}=\frac{B_{m} C_{m}-D_{m} E_{m}}{A_{m} B_{m}-E_{m}^{2}}, \\
& M_{2(\mathrm{opt})}=\frac{A_{m} D_{m}-C_{m} E_{m}}{A_{m} B_{m}-E_{m}^{2}} .
\end{aligned}
$$

The minimum MSE of $\widehat{\bar{Y}}_{(M)}^{\left(\alpha_{1}, \alpha_{2}, \alpha_{3}, \gamma_{1}, \gamma_{2}, \gamma_{3}\right)}$ to the first order of approximation is given by

$$
\operatorname{MSE}\left(\hat{\bar{Y}}_{(M)}^{\left(\alpha_{1}, \alpha_{2}, \alpha_{3}, \gamma_{1}, \gamma_{2}, \gamma_{3}\right)}\right)_{\min } \cong \bar{Y}^{2}\left[1-\frac{A_{m} D_{m}^{2}+B_{m} C_{m}^{2}-2 C_{m} D_{m} E_{m}}{A_{m} B_{m}-E_{m}^{2}}\right]
$$

Some special estimators as members of the proposed general class of estimators are given by

$$
\widehat{\bar{Y}}_{(M)}^{(1,0,0,1,0,0)}=\bar{y}\left[M_{1}\left\{\frac{\bar{X}^{*}}{\bar{x}^{*}}\right\}+M_{2}\left\{\frac{\bar{Z}^{*}}{\bar{z}^{*}}\right\}\right] .
$$

(i) Putting $\alpha_{1}=\gamma_{1}=1, \alpha_{2}=\alpha_{3}=\gamma_{2}=\gamma_{3}=0$ in (25), we get

(ii) Putting $\alpha_{2}=\gamma_{2}=1, \alpha_{1}=\alpha_{3}=\gamma_{1}=\gamma_{3}=0$ in (25), we get

$$
\widehat{\bar{Y}}_{(M)}^{(0,1,0,0,1,0)}=\bar{y}\left[M_{1}\left\{\exp \left(\frac{\bar{X}^{*}-\bar{x}^{*}}{\bar{X}^{*}+\bar{x}^{*}}\right)\right\}+M_{2}\left\{\exp \left(\frac{\bar{Z}^{*}-\bar{z}^{*}}{\bar{Z}^{*}+\bar{z}^{*}}\right)\right\}\right] .
$$

(iii) Putting $\alpha_{3}=\gamma_{3}=1, \alpha_{1}=\alpha_{2}=\gamma_{1}=\gamma_{2}=0$ in (25), we get

$$
\widehat{\bar{Y}}_{(M)}^{(0,0,1,0,0,1)}=\bar{y}\left[M_{1}\left\{1+\log \left(\frac{\bar{X}^{*}}{\bar{x}^{*}}\right)\right\}+M_{2}\left\{1+\log \left(\frac{\bar{Z}^{*}}{\bar{z}^{*}}\right)\right\}\right] .
$$

(iv) Putting $\alpha_{1}=\alpha_{2}=\gamma_{2}=1, \alpha_{3}=\gamma_{1}=\gamma_{3}=0$ in (25), we get

$$
\hat{\bar{Y}}_{(M)}^{(1,1,0,0,1,0)}=\bar{y}\left[M_{1}\left\{\frac{\bar{X}^{*}}{\bar{x}^{*}}\right\}\left\{\exp \left(\frac{\bar{X}^{*}-\bar{x}^{*}}{\bar{X}^{*}+\bar{x}^{*}}\right)\right\}+M_{2}\left\{\exp \left(\frac{\bar{Z}^{*}-\bar{z}^{*}}{\bar{Z}^{*}+\bar{z}^{*}}\right)\right\}\right] .
$$

(v) Putting $\alpha_{1}=\alpha_{3}=\gamma_{2}=1, \alpha_{2}=\gamma_{1}=\gamma_{3}=0$ in (25), we get

$$
\hat{\bar{Y}}_{(M)}^{(1,0,1,0,1,0)}=\bar{y}\left[M_{1}\left\{\frac{\bar{X}^{*}}{\bar{x}^{*}}\right\}\left\{1+\log \left(\frac{\bar{X}^{*}}{\bar{x}^{*}}\right)\right\}+M_{2}\left\{\exp \left(\frac{\bar{Z}^{*}-\bar{z}^{*}}{\bar{Z}^{*}+\bar{z}^{*}}\right)\right\}\right] .
$$


(vi) Putting $\alpha_{2}=\alpha_{3}=\gamma_{2}=1, \alpha_{1}=\gamma_{1}=\gamma_{3}=0$ in (25), we get

$$
\hat{\bar{Y}}_{(M)}^{(0,1,1,0,1,0)}=\bar{y}\left[M_{1}\left\{\exp \left(\frac{\bar{X}^{*}-\bar{x}^{*}}{\bar{X}^{*}+\bar{x}^{*}}\right)\right\}\left\{1+\log \left(\frac{\bar{X}^{*}}{\bar{x}^{*}}\right)\right\}+M_{2}\left\{\exp \left(\frac{\bar{Z}^{*}-\bar{z}^{*}}{\bar{Z}^{*}+\bar{z}^{*}}\right)\right\}\right] .
$$

(vii) Putting $\alpha_{1}=\alpha_{2}=\alpha_{3}=\gamma_{2}=1, \gamma_{1}=\gamma_{3}=0$ in (25), we get

$$
\hat{\bar{Y}}_{(M)}^{(1,1,1,0,1,0)}=\bar{y}\left[M_{1}\left\{\frac{\bar{X}^{*}}{\bar{x}^{*}}\right\}\left\{\exp \left(\frac{\bar{X}^{*}-\bar{x}^{*}}{\bar{X}^{*}+\bar{x}^{*}}\right)\right\}\left\{1+\log \left(\frac{\bar{X}^{*}}{\bar{x}^{*}}\right)\right\}+M_{2}\left\{\exp \left(\frac{\bar{Z}^{*}-\bar{z}^{*}}{\bar{Z}^{*}+\bar{z}^{*}}\right)\right\}\right] .
$$

(viii) Putting $\alpha_{1}=\gamma_{1}=\gamma_{2}=\gamma_{3}=1, \alpha_{2}=\alpha_{3}=0$ in (25), we get

$$
\hat{\bar{Y}}_{(M)}^{(1,0,0,1,1,1)}=\bar{y}\left[M_{1}\left\{\frac{\bar{X}^{*}}{\bar{x}^{*}}\right\}+M_{2}\left\{\frac{\bar{Z}^{*}}{\bar{z}^{*}}\right\}\left\{\exp \left(\frac{\bar{Z}^{*}-\bar{z}^{*}}{\bar{Z}^{*}+\bar{z}^{*}}\right)\right\}\left\{1+\log \left(\frac{\bar{Z}^{*}}{\bar{z}^{*}}\right)\right\}\right] .
$$

(i) Putting $\alpha_{2}=\gamma_{1}=\gamma_{2}=\gamma_{3}=1, \alpha_{1}=\alpha_{3}=0$ in (25), we get

$$
\hat{\bar{Y}}_{(M)}^{(0,1,0,1,1,1)}=\bar{y}\left[M_{1}\left\{\exp \left(\frac{\bar{X}^{*}-\bar{x}^{*}}{\bar{X}^{*}+\bar{x}^{*}}\right)\right\}+M_{2}\left\{\frac{\bar{Z}^{*}}{\bar{z}^{*}}\right\}\left\{\exp \left(\frac{\bar{Z}^{*}-\bar{z}^{*}}{\bar{Z}^{*}+\bar{z}^{*}}\right)\right\}\left\{1+\log \left(\frac{\bar{Z}^{*}}{\bar{z}^{*}}\right)\right\}\right] .
$$

(x) Putting $\alpha_{3}=\gamma_{1}=\gamma_{2}=\gamma_{3}=1, \alpha_{1}=\alpha_{2}=0$ in (25), we get

$$
\hat{\bar{Y}}_{(M)}^{(0,0,1,1,1,1)}=\bar{y}\left[M_{1}\left\{1+\log \left(\frac{\bar{X}^{*}}{\bar{x}^{*}}\right)\right\}+M_{2}\left\{\frac{\bar{Z}^{*}}{\bar{z}^{*}}\right\}\left\{\exp \left(\frac{\bar{Z}^{*}-\bar{z}^{*}}{\bar{Z}^{*}+\bar{z}^{*}}\right)\right\}\left\{1+\log \left(\frac{\bar{Z}^{*}}{\bar{z}^{*}}\right)\right\}\right]
$$


Note: We can generate more sub-classes of the proposed general class of estimators by using different combinations.

\section{Numerical Example}

We use the following four real data sets for a numerical study.

Population 1 (see [19]):

$$
\begin{aligned}
Y & =\text { Area under wheat in acres in } 1974, \\
X & =\text { Area under wheat in acres in } 1971, \\
Z & =\text { Area under wheat in acres in } 1973, \\
N & =34, \\
n & =20, \\
\bar{Y} & =856.412, \\
\bar{X} & =208.882, \\
\bar{Z} & =199.441, \\
C_{y} & =0.8561, \\
C_{x} & =0.721, \\
C_{z} & =0.753, \\
\rho_{y x} & =0.449, \\
\rho_{y z} & =0.443, \\
\rho_{x z} & =0.980, \\
\beta_{2 x} & =2.910, \\
\beta_{2 z} & =3.732 .
\end{aligned}
$$

Population 2 (see [18]):

$$
\begin{aligned}
Y & =\text { Output of the factory, } \\
X & =\text { Number of workers, } \\
Z & =\text { Fixed capital, } \\
N & =80, \\
n & =20 \\
\bar{Y} & =5182.637, \\
\bar{X} & =285.125 \\
\bar{Z} & =1126.463, \\
C_{y} & =0.354 \\
C_{x} & =0.948 \\
C_{z} & =0.751 \\
\rho_{y x} & =0.915 \\
\rho_{y z} & =0.941 \\
\rho_{x z} & =0.988 \\
\beta_{2 x} & =0.698 \\
\beta_{2 z} & =1.050 .
\end{aligned}
$$

Population 3 (Punjab Development Statistics (2019)): This data is taken from Punjab development of statistics of 36 districts of Punjab, Pakistan during 2018.

$$
\begin{aligned}
Y & =\text { Number of reported crimes by hurt, } \\
X & =\text { Number of reported crimes by murdered, } \\
Z & =\text { Number of reported crimes by kidnapped, } \\
N & =36, \\
n & =10, \\
\bar{Y} & =421.9722, \\
\bar{X} & =112.3889, \\
\bar{Z} & =412.2222, \\
C_{y} & =0.5718, \\
C_{x} & =0.8336, \\
C_{z} & =1.4229, \\
\rho_{y x} & =0.7786, \\
\rho_{y z} & =0.6900, \\
\rho_{x z} & =0.8483, \\
\beta_{2 x} & =7.5508, \\
\beta_{2 z} & =27.5415 .
\end{aligned}
$$

Population 4 (see [19]):

This data are based on 69 villages of Doraha development bloc of Punjab, India.

$$
\begin{aligned}
Y & =\text { Number of tube wells, } \\
X & =\text { Number of tractors, } \\
Z & =\text { Net irrigated area in hectares, } \\
N & =69 \\
n & =10 \\
\bar{Y} & =135.2609 \\
\bar{X} & =21.2319 \\
\bar{Z} & =345.7536 \\
C_{y} & =0.8422 \\
C_{x} & =0.7969 \\
C_{z} & =0.8478 \\
\rho_{y x} & =0.9118 \\
\rho_{y z} & =0.9224 \\
\rho_{x z} & =0.9007 \\
\beta_{2 x} & =3.7653 \\
\beta_{2 z} & =7.2159
\end{aligned}
$$


The results based on Populations 1-4 are given in Tables 1-11. We use the following expression to obtain the percent relative efficiency (PRE) as

$$
\text { PRE }=\frac{\operatorname{Var}\left(\hat{\bar{Y}}_{(0)}\right)}{\operatorname{MSE}(.)} \times 100,
$$

where $()=.\hat{\bar{Y}}_{(0)}, \hat{\bar{Y}}_{(R)}^{(i)}, \quad \hat{\bar{Y}}_{(E)}^{(i)}, \hat{\bar{Y}}_{(\mathrm{log})}^{(i)}, \hat{\bar{Y}}_{(\mathrm{reg})}^{(i)} \hat{\bar{Y}}_{(\mathrm{reg})}^{(\mathrm{j})}(i=1,2,3$; $j=M, S, U)$.

In Table 1, we observed the following:

(i) The ratio and log-ratio estimators $\left(\hat{\bar{Y}}_{(R)}^{(1)}, \hat{\bar{Y}}_{(\log )}^{(1)}\right)$ in population 2, $\left(\hat{\bar{Y}}_{(R)}^{(2)}, \hat{\bar{Y}}_{(\log )}^{(2)}\right)$ in populations 2 and 3, and $\left(\hat{\bar{Y}}_{(R)}^{(3)}, \hat{\bar{Y}}_{(\log )}^{(3)}\right)$ in all four populations are performing poorly as compared to $\hat{\bar{Y}}_{(0)}$.

(ii) The exponential-ratio estimator $\hat{\bar{Y}}_{(E)}^{(3)}$ in Populations 2 and 3 is not performing good.

(iii) Mohanty [2] regression estimator $\hat{\bar{Y}}_{(\mathrm{Reg})}^{(M)}$ in populations 1-3 and Swain [5] estimator $\hat{\bar{Y}}_{(\mathrm{Reg})}^{(S)}$ in Population 3 are not efficient as compared to $\widehat{\bar{Y}}_{(0)}$.

(iv) Among all the estimators above discussed, the performance of $\hat{\bar{Y}}_{(\mathrm{Reg})}^{(U)}$ is the best.

\section{Comparison of Estimators}

Now we compare the proposed class of estimators with other existing estimators.

Condition 1. By (2) and (33), $\operatorname{MSE}\left(\hat{\bar{Y}}_{(M)}^{\left(\alpha_{1}, \alpha_{2}, \alpha_{3}, \gamma_{1}, \gamma_{2}, \gamma_{3}\right)}\right)_{\min }<$ $\operatorname{Var}\left(\overline{\bar{Y}}_{(0)}\right)$ if

$$
\left[\Theta C_{y}^{2}+\frac{O_{1}}{O_{2}}-1\right]>0
$$

where $O_{1}=A_{m} D_{m}^{2}+B_{m} C_{m}^{2}-2 C_{m} D_{m} E_{m}$ and $O_{2}=A_{m} B_{m}^{-}$ $E_{m}^{2}$.

Condition 2. By ((4)-(6)) or ((12)-(14)) and (33), $\left.\left.\operatorname{MSE}\left(\hat{\bar{Y}}_{(M)}^{\left(\alpha_{1}, \alpha_{2}, \alpha_{3}, \gamma_{1}, \gamma_{2},\right.} \gamma_{3}\right)\right)_{\min }<\operatorname{MSE}\left(\hat{\bar{Y}}_{(R)}^{(i)}\right), \operatorname{MSE}\left(\hat{\bar{Y}}_{(\log )}^{(i)}\right)\right](i=$ $1,2,3)$ if

$$
\begin{array}{r}
{\left[\Theta\left\{C_{y}^{2}+C_{x}^{2}-2 C_{y x}\right\}+\frac{O_{1}}{O_{2}}-1\right]>0,} \\
{\left[\Theta\left\{C_{y}^{2}+C_{z}^{2}-2 C_{y z}\right\}+\frac{O_{1}}{O_{2}}-1\right]>0,} \\
{\left[\Theta\left\{C_{y}^{2}+C_{x}^{2}+C_{z}^{2}-2\left(C_{y x}+C_{y z}\right)+2 C_{x z}\right\}+\frac{O_{1}}{O_{2}}-1\right]>0 .}
\end{array}
$$

\begin{tabular}{|c|c|c|c|c|}
\hline Estimator & Pop.1 & Pop. 2 & Pop. 3 & Pop. 4 \\
\hline$\widehat{\bar{Y}}_{(0)}$ & 100.00 & 100.00 & 100.00 & 100.00 \\
\hline$\widehat{\bar{Y}}_{(R)}^{(1)}, \widehat{\bar{Y}}_{(\log )}^{(1)}$ & 104.932 & $*$ & 116.936 & 588.911 \\
\hline$\hat{\bar{Y}}_{(R)}^{(2)}, \widehat{\bar{Y}}_{(\log )}^{(2)}$ & 100.926 & $*$ & $*$ & 639.893 \\
\hline$\widehat{\bar{Y}}_{(R)}^{(3)}, \widehat{\bar{Y}}_{(\log )}^{(3)}$ & $*$ & $*$ & $*$ & $*$ \\
\hline$\widehat{\bar{Y}}_{(E)}^{(1)}$ & 125.129 & 291.940 & 252.366 & 276.952 \\
\hline$\widehat{\bar{Y}}_{(E)}^{(2)}$ & 124.688 & 776.064 & 120.327 & 307.879 \\
\hline$\widehat{\bar{Y}}_{(E)}^{(3)}$ & 103.718 & $*$ & $*$ & 870.810 \\
\hline$\hat{\bar{Y}}_{(\operatorname{Reg})}^{(1)}$ & 125.251 & 614.345 & 253.948 & 593.047 \\
\hline$\widehat{\bar{Y}}_{(\mathrm{Reg})}^{(2)}$ & 124.692 & 873.218 & 190.876 & 670.339 \\
\hline$\widehat{\bar{Y}}_{(\text {Reg })}^{(3)}$ & 100.807 & 102.181 & 120.605 & 120.069 \\
\hline$\hat{\bar{Y}}_{(\mathrm{Reg})}^{(M)}$ & $*$ & $*$ & $*$ & 102.214 \\
\hline$\widehat{\bar{Y}}_{(\operatorname{Reg})}^{(S)}$ & 121.910 & 883.094 & $*$ & 640.000 \\
\hline$\widehat{\bar{Y}}_{(\mathrm{Reg})}^{(U)}$ & 125.349 & 948.307 & 255.867 & 873.966 \\
\hline
\end{tabular}

TABLE 1: PRE of different estimators with respect $\hat{\bar{Y}}_{(0)}$ when $(a=c=1, b=d=0)$.

TABLE 2: PRE of proposed estimator when $\left(\alpha_{1}=\gamma_{1}=1, \alpha_{2}=\alpha_{3}=\gamma_{2}=\gamma_{3}=0\right)$.

\begin{tabular}{lccccccc}
\hline$a$ & $b$ & $c$ & $d$ & Pop. 1 & Pop. 2 & Pop. 3 & Pop. 4 \\
\hline 1 & 0 & 1 & 0 & 107.697 & 186.585 & 132.429 & 886.815 \\
1 & $C_{x}$ & 1 & $C_{z}$ & 107.934 & 184.610 & 134.467 & 885.501 \\
$\beta_{2 x}$ & $C_{x}$ & $\beta_{2 z}$ & $C_{z}$ & 107.780 & 183.288 & 132.707 & 886.865 \\
$C_{x}$ & $\beta_{2 x}$ & $C_{z}$ & $\beta_{2 z}$ & 107.780 & 183.288 & 132.707 & 886.865 \\
1 & $\rho_{y x}$ & 1 & $\rho_{y z}$ & 107.780 & 183.288 & 132.707 & 886.865 \\
1 & $\beta_{2 x}$ & 1 & $\beta_{2 z}$ & 108.628 & 185.787 & 149.748 & 852.370 \\
$C_{x}$ & $\rho_{y x}$ & $C_{z}$ & $\rho_{y z}$ & 107.903 & 185.219 & 134.781 & 883.828 \\
$\rho_{y x}$ & $C_{x}$ & $\rho_{y z}$ & $C_{z}$ & 108.220 & 184.398 & 135.032 & 885.176 \\
$\beta_{2 x}$ & $\rho_{y z}$ & $\beta_{2 z}$ & $\rho_{y z}$ & 107.749 & 183.672 & 132.690 & 886.850 \\
$\rho_{y x}$ & $\beta_{2 x}$ & $\rho_{y z}$ & $\beta_{2 z}$ & 109.745 & 185.671 & 154.632 & 846.972 \\
\hline
\end{tabular}

$$
\begin{array}{r}
{\left[\Theta\left\{C_{y}^{2}+\frac{1}{4} C_{x}^{2}-C_{y x}\right\}+\frac{O_{1}}{O_{2}}-1\right]>0,} \\
{\left[\Theta\left\{C_{y}^{2}+\frac{1}{4} C_{z}^{2}-C_{y z}\right\}+\frac{O_{1}}{O_{2}}-1\right]>0,} \\
{\left[\Theta\left\{C_{y}^{2}+\frac{1}{4}\left(C_{x}^{2}+C_{z}^{2}\right)-\left(C_{y x}+C_{y z}\right)+\frac{1}{2} C_{x z}\right\}+\frac{O_{1}}{O_{2}}-1\right]>0 .}
\end{array}
$$

$\begin{array}{llll}\text { Condition 3. By } \quad((8)-(10)) \quad \text { and } & \text { (33), } & \operatorname{MSE} & \text { Condition 4. By } \quad((16)-(18)) \quad \text { and } \\ \left(\hat{\bar{Y}}_{(M)}^{\left(\alpha_{1}, \alpha_{2}, \alpha_{3}, \gamma_{1}, \gamma_{2}, \gamma_{3}\right)}\right)_{\min }<\operatorname{MSE}\left(\hat{\bar{Y}}_{(E)}^{(i)}\right)(i=1,2,3) \text { if } & & \operatorname{MSE}\left(\hat{\bar{Y}}_{(M)}^{\left(\alpha_{1}, \alpha_{2}, \alpha_{3}, \gamma_{1}, \gamma_{2}, \gamma_{3}\right)}\right)_{\min }<\operatorname{MSE}\left(\hat{\bar{Y}}_{(\operatorname{Reg})}^{(i)}\right)(i=1,2,3) \text { if }\end{array}$ 
TABLE 3: PRE of proposed estimator when $\left(\alpha_{2}=\gamma_{2}=1, \alpha_{1}=\alpha_{3}=\gamma_{1}=\gamma_{3}=0\right)$.

\begin{tabular}{|c|c|c|c|c|c|c|c|}
\hline$a$ & $b$ & $c$ & $d$ & Pop. 1 & Pop. 2 & Pop. 3 & Pop. 4 \\
\hline 1 & 0 & 1 & 0 & 127.090 & 956.748 & 264.876 & 311.868 \\
\hline 1 & $C_{x}$ & 1 & $C_{z}$ & 127.073 & 956.817 & 264.643 & 310.282 \\
\hline$\beta_{2 x}$ & $C_{x}$ & $\beta_{2 z}$ & $C_{z}$ & 127.084 & 956.854 & 264.849 & 311.339 \\
\hline$C_{x}$ & $\beta_{2 x}$ & $C_{z}$ & $\beta_{2 z}$ & 126.980 & 956.775 & 261.317 & 319.839 \\
\hline 1 & $\rho_{y x}$ & 1 & $\rho_{y z}$ & 127.079 & 956.808 & 264.675 & 310.291 \\
\hline 1 & $\beta_{2 x}$ & 1 & $\beta_{2 z}$ & 127.011 & 956.783 & 261.624 & 314.469 \\
\hline$C_{x}$ & $\rho_{y x}$ & $C_{z}$ & $\rho_{y z}$ & 127.075 & 956.803 & 264.642 & 310.422 \\
\hline$\rho_{y x}$ & $C_{x}$ & $\rho_{y z}$ & $C_{z}$ & 127.051 & 956.824 & 264.568 & 310.257 \\
\hline$\beta_{2 x}$ & $\rho_{y z}$ & $\beta_{2 z}$ & $\rho_{y z}$ & 127.086 & 956.846 & 264.851 & 311.285 \\
\hline$\rho_{y x}$ & $\beta_{2 x}$ & $\rho_{y z}$ & $\beta_{2 z}$ & 126.900 & 956.788 & 260.260 & 316.213 \\
\hline
\end{tabular}

TABLE 4: PRE of proposed estimator when $\left(\alpha_{3}=\gamma_{3}=1, \alpha_{1}=\alpha_{2}=\gamma_{1}=\gamma_{2}=0\right)$.

\begin{tabular}{|c|c|c|c|c|c|c|c|}
\hline$a$ & $b$ & $c$ & $d$ & Pop. 1 & Pop. 2 & Pop. 3 & Pop. 4 \\
\hline 1 & 0 & 1 & 0 & 106.402 & 186.926 & 127.151 & 919.696 \\
\hline 1 & $C_{x}$ & 1 & $C_{z}$ & 106.646 & 184.941 & 129.254 & 921.254 \\
\hline$\beta_{2 x}$ & $C_{x}^{x}$ & $\beta_{2 z}$ & $C_{z}$ & 106.488 & 183.610 & 127.443 & 920.619 \\
\hline$C_{x}$ & $\beta_{2 x}^{x}$ & $C_{z}$ & $\beta_{2 z}$ & 107.724 & 186.467 & 149.441 & 873.745 \\
\hline 1 & $\rho_{y x}$ & 1 & $\rho_{y z}$ & 106.555 & 185.296 & 129.179 & 921.052 \\
\hline 1 & $\beta_{2 x}$ & 1 & $\beta_{2 z}$ & 107.357 & 186.124 & 144.723 & 889.198 \\
\hline$C_{x}$ & $\rho_{y x}$ & $C_{z}$ & $\rho_{y z}$ & 106.615 & 185.553 & 129.618 & 920.360 \\
\hline$\rho_{y x}$ & $C_{x}$ & $\rho_{y z}$ & $C_{z}$ & 106.941 & 184.727 & 129.828 & 921.127 \\
\hline$\beta_{2 x}$ & $\rho_{y z}$ & $\beta_{2 z}$ & $\rho_{y z}$ & 106.456 & 183.997 & 127.426 & 920.719 \\
\hline$\rho_{y x}$ & $\beta_{2 x}$ & $\rho_{y z}$ & $\beta_{2 z}$ & 108.505 & 186.007 & 149.286 & 883.271 \\
\hline
\end{tabular}

TABLE 5: PRE of proposed estimator when $\left(\alpha_{1}=\alpha_{2}=\gamma_{2}=1, \alpha_{3}=\gamma_{1}=\gamma_{3}=0\right)$.

\begin{tabular}{|c|c|c|c|c|c|c|c|}
\hline$a$ & $b$ & $c$ & $d$ & Pop. 1 & Pop. 2 & Pop. 3 & Pop. 4 \\
\hline 1 & 0 & 1 & 0 & 126.769 & 914.223 & 141.181 & 807.850 \\
\hline 1 & $C_{x}$ & 1 & $C_{z}$ & 126.777 & 914.152 & 141.896 & 794.946 \\
\hline$\beta_{2 x}$ & $C_{x}^{x}$ & $\beta_{2 z}$ & $C_{z}$ & 126.771 & 914.197 & 141.216 & 804.456 \\
\hline$C_{x}$ & $\beta_{2 x}^{x}$ & $C_{z}$ & $\beta_{2 z}^{2}$ & 126.818 & 913.997 & 150.724 & 732.960 \\
\hline 1 & $\rho_{y x}$ & 1 & $\rho_{y z}$ & 126.774 & 914.109 & 141.569 & 793.158 \\
\hline 1 & $\beta_{2 x}$ & 1 & $\beta_{2 z}$ & 126.807 & 914.066 & 153.920 & 747.374 \\
\hline$C_{x}$ & $\rho_{y x}$ & $C_{z}$ & $\rho_{y z}$ & 126.775 & 914.048 & 141.497 & 789.553 \\
\hline$\rho_{y x}$ & $C_{x}$ & $\rho_{y z}$ & $C_{z}$ & 126.786 & 914.150 & 142.202 & 793.730 \\
\hline$\beta_{2 x}$ & $\rho_{y z}$ & $\beta_{2 z}$ & $\rho_{y z}$ & 126.770 & 914.155 & 141.204 & 803.975 \\
\hline$\rho_{y x}$ & $\beta_{2 x}$ & $\rho_{y z}$ & $\beta_{2 z}$ & 126.849 & 914.058 & 159.313 & 741.821 \\
\hline
\end{tabular}

TABLE 6: PRE of proposed estimator when $\left(\alpha_{1}=\alpha_{3}=\gamma_{2}=1, \alpha_{2}=\gamma_{1}=\gamma_{3}=0\right)$.

\begin{tabular}{|c|c|c|c|c|c|c|c|}
\hline$a$ & $b$ & $c$ & $d$ & Pop. 1 & Pop. 2 & Pop. 3 & Pop. 4 \\
\hline 1 & 0 & 1 & 0 & 126.737 & 912.457 & 139.553 & 845.147 \\
\hline 1 & $C_{x}$ & 1 & $C_{z}$ & 126.743 & 912.383 & 140.132 & 836.605 \\
\hline$\beta_{2 x}$ & $C_{x}$ & $\beta_{2 z}$ & $\mathrm{C}_{z}$ & 126.739 & 912.423 & 139.573 & 842.896 \\
\hline$C_{x}$ & $\beta_{2 x}$ & $C_{z}$ & $\beta_{2 z}$ & 126.779 & 912.237 & 147.425 & 797.474 \\
\hline 1 & $\rho_{y x}$ & 1 & $\rho_{y z}$ & 126.741 & 912.342 & 139.830 & 835.452 \\
\hline 1 & $\beta_{2 x}$ & 1 & $\beta_{2 z}$ & 126.769 & 912.303 & 150.655 & 806.104 \\
\hline$C_{x}$ & $\rho_{y x}$ & $C_{z}$ & $\rho_{y z}$ & 126.742 & 914.284 & 139.744 & 833.140 \\
\hline$\rho_{y x}$ & $C_{x}$ & $\rho_{y z}$ & $C_{z}$ & 126.752 & 912.380 & 140.393 & 835.815 \\
\hline$\beta_{2 x}$ & $\rho_{y z}$ & $\beta_{2 z}$ & $\rho_{y z}$ & 126.738 & 914.383 & 139.562 & 842.580 \\
\hline$\rho_{y x}$ & $\beta_{2 x}$ & $\rho_{y z}$ & $\beta_{2 z}$ & 126.805 & 912.295 & 155.475 & 802.724 \\
\hline
\end{tabular}


TABLE 7: PRE of proposed estimator when $\left(\alpha_{2}=\alpha_{3}=\gamma_{2}=1, \alpha_{1}=\gamma_{1}=\gamma_{3}=0\right)$.

\begin{tabular}{|c|c|c|c|c|c|c|c|}
\hline$a$ & $b$ & $c$ & $d$ & Pop. 1 & Pop. 2 & Pop. 3 & Pop. 4 \\
\hline 1 & 0 & 1 & 0 & 126.710 & 920.174 & 140.021 & 784.443 \\
\hline 1 & $C_{x}$ & 1 & $C_{z}$ & 126.715 & 920.076 & 140.691 & 775.612 \\
\hline$\beta_{2 x}$ & $\hat{C_{x}}$ & $\beta_{2 z}$ & $C_{z}$ & 126.711 & 920.126 & 140.050 & 782.185 \\
\hline$C_{x}$ & $\beta_{2 x}$ & $C_{z}$ & $\beta_{2 z}$ & 126.743 & 919.891 & 149.088 & 727.261 \\
\hline 1 & $\rho_{y x}$ & 1 & $\rho_{y z}$ & 126.713 & 920.025 & 140.371 & 774.362 \\
\hline 1 & $\beta_{2 x}$ & 1 & $\beta_{2 z}$ & 126.735 & 919.976 & 152.300 & 739.153 \\
\hline$C_{x}$ & $\rho_{y x}$ & $C_{z}$ & $\rho_{y z}$ & 126.714 & 919.950 & 140.293 & 771.816 \\
\hline$\rho_{y x}$ & $C_{x}$ & $\rho_{y z}$ & $C_{z}$ & 126.721 & 920.073 & 140.983 & 774.759 \\
\hline$\beta_{2 x}$ & $\rho_{y z}$ & $\beta_{2 z}$ & $\rho_{y z}$ & 126.710 & 920.075 & 140.038 & 781.863 \\
\hline$\rho_{y x}$ & $\beta_{2 x}$ & $\rho_{y z}$ & $\beta_{2 z}$ & 126.763 & 919.965 & 157.553 & 734.610 \\
\hline
\end{tabular}

TABLE 8: PRE of proposed estimator when $\left(\alpha_{1}=\alpha_{2}=\alpha_{3}=\gamma_{2}=1, \gamma_{1}=\gamma_{3}=0\right)$.

\begin{tabular}{|c|c|c|c|c|c|c|c|}
\hline$a$ & $b$ & $c$ & $d$ & Pop. 1 & Pop. 2 & Pop. 3 & Pop. 4 \\
\hline 1 & 0 & 1 & 0 & 126.768 & 907.889 & 139.833 & 902.503 \\
\hline 1 & $C_{x}$ & 1 & $C_{z}$ & 126.776 & 907.822 & 140.396 & 890.351 \\
\hline$\beta_{2 x}$ & $C_{x}$ & $\beta_{2 z}$ & $C_{z}$ & 126.770 & 907.856 & 139.852 & 899.195 \\
\hline$C_{x}$ & $\beta_{2 x}^{x}$ & $C_{z}$ & $\beta_{2 z}$ & 126.818 & 907.695 & 147.416 & 843.179 \\
\hline 1 & $\rho_{y x}$ & 1 & $\rho_{y z}$ & 126.772 & 907.787 & 140.103 & 888.744 \\
\hline 1 & $\beta_{2 x}$ & 1 & $\beta_{2 z}$ & 126.806 & 907.753 & 150.567 & 852.694 \\
\hline$C_{x}$ & $\rho_{y x}$ & $C_{z}$ & $\rho_{y z}$ & 126.774 & 907.736 & 140.019 & 885.566 \\
\hline$\rho_{y x}$ & $C_{x}$ & $\rho_{y z}$ & $C_{z}$ & 126.785 & 907.820 & 140.649 & 889.257 \\
\hline$\beta_{2 x}$ & $\rho_{y z}$ & $\beta_{2 z}$ & $\rho_{y z}$ & 126.769 & 907.821 & 139.842 & 898.731 \\
\hline$\rho_{y x}$ & $\beta_{2 x}$ & $\rho_{y z}$ & $\beta_{2 z}$ & 126.850 & 907.746 & 155.212 & 848.913 \\
\hline
\end{tabular}

TABLE 9: PRE of proposed estimator when $\left(\alpha_{1}=\gamma_{1}=\gamma_{2}=\gamma_{3}=1, \alpha_{2}=\alpha_{3}=0\right)$.

\begin{tabular}{|c|c|c|c|c|c|c|c|}
\hline$a$ & $b$ & $c$ & $d$ & Pop. 1 & Pop. 2 & Pop. 3 & Pop. 4 \\
\hline 1 & 0 & 1 & 0 & 123.139 & 145.452 & 159.789 & 669.527 \\
\hline 1 & $C_{x}$ & 1 & $C_{z}$ & 123.185 & 146.707 & 161.602 & 702.708 \\
\hline$\beta_{2 x}$ & $C_{x}^{x}$ & $\beta_{2 z}$ & $C_{z}$ & 123.155 & 147.296 & 160.040 & 678.643 \\
\hline$C_{x}$ & $\beta_{2 x}^{x}$ & $C_{z}$ & $\beta_{2 z}$ & 123.384 & 146.324 & 178.354 & 820.621 \\
\hline 1 & $\rho_{y x}$ & 1 & $\rho_{y z}$ & 123.168 & 146.636 & 161.528 & 707.218 \\
\hline 1 & $\beta_{2 x}$ & 1 & $\beta_{2 z}$ & 123.317 & 146.313 & 174.823 & 798.447 \\
\hline$C_{x}$ & $\rho_{y x}$ & $C_{z}$ & $\rho_{y z}$ & 123.179 & 146.668 & 161.898 & 716.133 \\
\hline$\rho_{y x}$ & $C_{x}$ & $\rho_{y z}$ & $C_{z}$ & 123.240 & 146.826 & 162.099 & 705.743 \\
\hline$\beta_{2 x}$ & $\rho_{y z}$ & $\beta_{2 z}$ & $\rho_{y z}$ & 123.149 & 147.206 & 160.025 & 679.938 \\
\hline$\rho_{y x}$ & $\beta_{2 x}$ & $\rho_{y z}$ & $\beta_{2 z}$ & 123.523 & 146.397 & 178.726 & 807.318 \\
\hline
\end{tabular}

TABLE 10: PRE of proposed estimator when $\left(\alpha_{2}=\gamma_{1}=\gamma_{2}=\gamma_{3}=1, \alpha_{1}=\alpha_{3}=0\right)$.

\begin{tabular}{|c|c|c|c|c|c|c|c|}
\hline$a$ & $b$ & $c$ & $d$ & Pop. 1 & Pop. 2 & Pop. 3 & Pop. 4 \\
\hline 1 & 0 & 1 & 0 & 127.304 & 483.947 & 272.583 & 956.165 \\
\hline 1 & $C_{x}$ & 1 & $C_{z}$ & 127.306 & 485.394 & 272.843 & 952.519 \\
\hline$\beta_{2 x}$ & $C_{x}$ & $\beta_{2 z}$ & $\mathrm{C}_{z}$ & 127.304 & 486.023 & 272.627 & 955.357 \\
\hline$C_{x}$ & $\beta_{2 x}$ & $C_{z}$ & $\beta_{2 z}$ & 127.314 & 485.060 & 274.208 & 928.019 \\
\hline 1 & $\rho_{y x}$ & 1 & $\rho_{y z}$ & 127.305 & 485.341 & 272.869 & 952.032 \\
\hline 1 & $\beta_{2 x}$ & 1 & $\beta_{2 z}$ & 127.311 & 485.006 & 273.258 & 933.369 \\
\hline$C_{x}$ & $\rho_{y x}$ & $C_{z}$ & $\rho_{y z}$ & 127.306 & 485.413 & 272.947 & 952.016 \\
\hline$\rho_{y x}$ & $C_{x}$ & $\rho_{y z}$ & $C_{z}$ & 127.308 & 485.528 & 272.898 & 952.166 \\
\hline$\beta_{2 x}$ & $\rho_{y z}$ & $\beta_{2 z}$ & $\rho_{y z}$ & 127.304 & 485.948 & 272.626 & 955.247 \\
\hline$\rho_{y x}$ & $\beta_{2 x}$ & $\rho_{y z}$ & $\beta_{2 z}$ & 127.318 & 485.105 & 272.896 & 931.187 \\
\hline
\end{tabular}


TABLE 11: PRE of proposed estimator when $\left(\alpha_{3}=\gamma_{1}=\gamma_{2}=\gamma_{3}=1, \alpha_{1}=\alpha_{2}=0\right)$.

\begin{tabular}{|c|c|c|c|c|c|c|c|}
\hline$a$ & $b$ & $c$ & $d$ & Pop. 1 & Pop. 2 & Pop. 3 & Pop. 4 \\
\hline 1 & 0 & 1 & 0 & 122.150 & 178.926 & 180.747 & 649.616 \\
\hline 1 & $C_{x}$ & 1 & $C_{z}$ & 122.196 & 180.406 & 181.991 & 682.237 \\
\hline$\beta_{2 x}$ & $C_{x}$ & $\beta_{2 z}$ & $C_{z}$ & 122.167 & 181.159 & 180.961 & 658.386 \\
\hline$C_{x}$ & $\beta_{2 x}$ & $C_{z}$ & $\beta_{2 z}$ & 122.392 & 179.818 & 192.910 & 797.503 \\
\hline 1 & $\rho_{y x}$ & 1 & $\rho_{y z}$ & 122.179 & 180.286 & 182.139 & 686.675 \\
\hline 1 & $\beta_{2 x}$ & 1 & $\beta_{2 z}$ & 122.324 & 179.859 & 188.365 & 776.097 \\
\hline$C_{x}$ & $\rho_{y x}$ & $C_{z}$ & $\rho_{y z}$ & 122.191 & 180.278 & 182.524 & 695.431 \\
\hline$\rho_{y x}$ & $C_{x}$ & $\rho_{y z}$ & $C_{z}$ & 122.251 & 180.550 & 182.263 & 685.229 \\
\hline$\beta_{2 x}$ & $\rho_{y z}$ & $\beta_{2 z}$ & $\rho_{y z}$ & 122.161 & 181.016 & 180.955 & 659.670 \\
\hline$\rho_{y x}$ & $\beta_{2 x}$ & $\rho_{y z}$ & $\beta_{2 z}$ & 122.529 & 179.955 & 190.082 & 784.693 \\
\hline
\end{tabular}

$$
\begin{gathered}
{\left[\Theta C_{y}^{2}\left(1-\rho_{y x}^{2}\right)+\frac{O_{1}}{O_{2}}-1\right]>0,} \\
{\left[\Theta C_{y}^{2}\left(1-\rho_{y z}^{2}\right)+\frac{O_{1}}{O_{2}}-1\right]>0, \quad(52)} \\
{\left[\Theta C_{y}^{2}\left(1-\rho_{y x}^{2}-\rho_{y z}^{2}-2 \rho_{y x} \rho_{y z} \rho_{x z}\right)+\frac{O_{1}}{O_{2}}-1\right]>0 .} \\
\text { Condition } 5 . \text { By }(20) \text { and } \quad(38), \\
\left(\hat{\bar{Y}}_{(M)}^{\left(\alpha_{1}, \alpha_{2}, \alpha_{3}, \gamma_{1}, \gamma_{2}, \gamma_{3}\right)}\right)_{\min }<\operatorname{MSE}\left(\hat{\bar{Y}}_{(\mathrm{Reg})}^{(M)}\right) \text { if } \quad \mathrm{MSE} \\
{\left[\Theta\left\{C_{y}^{2}\left(1-\rho_{y x}^{2}\right)+C_{z}^{2}-2 C_{y z}+2 C_{y} C_{z} \rho_{y x} \rho_{x z}\right\}+\frac{O_{1}}{O_{2}}-1\right]>0 .}
\end{gathered}
$$

$$
\begin{aligned}
& \text { Condition 6. By } \quad(22) \text { and }(38), \quad \text { MSE } \\
& \left(\hat{\bar{Y}}_{(M)}^{\left(\alpha_{1}, \alpha_{2}, \alpha_{3}, \gamma_{1}, \gamma_{2}, \gamma_{3}\right)}\right)_{\min }<\operatorname{MSE}\left(\hat{\bar{Y}}_{(\mathrm{Reg})}^{(S)}\right)_{\min } \text { if } \\
& {\left[\Theta C_{y}^{2}\left\{\left(C_{y}^{2}+C_{z}^{2}-2 C_{y z}\right)-\left(C_{y} \rho_{y x}-C_{z} \rho_{x z}\right)^{2}\right\}+\frac{O_{1}}{O_{2}}-1\right]>0 .}
\end{aligned}
$$

$$
\begin{aligned}
& \text { Condition 7. By (32) and (38), MSE } \\
& \left(\hat{\bar{Y}}_{(M)}^{\left(\alpha_{1}, \alpha_{2}, \alpha_{3}, \gamma_{1}, \gamma_{2}, \gamma_{3}\right)}\right)_{\min }<\operatorname{MSE}\left(\widehat{\bar{Y}}_{(\mathrm{Reg})}^{(U)}\right)_{\min } \text { if } \\
& {\left[\Theta C_{y}^{2}\left(1-R_{y \cdot x z}^{2}\right)+\frac{O_{1}}{O_{2}}-1\right]>0 .}
\end{aligned}
$$

The proposed class of estimators will perform better when conditions 1-7 are satisfied.

\section{Conclusion}

In this study, we have proposed ratio-exponential-log type generalized class of estimators by combing a ratio, exponential-ratio, and log-ratio type estimators by using the linear transformation for finite population mean in simple random sampling. Expressions for the bias and MSE of proposed general class of estimators are obtained up to the first order of approximation. Four data sets are used for numerical study. Based on Tables 1-11, we observe that the proposed sub-classes of general estimators are performing well as compared to their competitor estimators. We have generated 10 sub-classes from the proposed general estimators with different combinations which all are efficient in different situation as compared to SRS. So, the proposed general class of estimators is preferable in further study.

\section{Data Availability}

The data used to support the findings of this study are included within the article.

\section{Conflicts of Interest}

The authors declare that they have no conflicts of interest.

\section{References}

[1] I. Olkin, "Multivariate ratio estimation for finite populations," Biometrika, vol. 45, no. 1-2, pp. 154-165, 1958.

[2] S. Mohanty, "Combination of regression and ratio estimate," Journal of Indian Statistical Association, vol. 5, pp. 16-19, 1967.

[3] W. A. Abu-Dayyeh, M. S. Ahmed, R. A. Ahmed, and H. A. Muttlak, "Some estimators of a finite population mean using auxiliary information," Applied Mathematics and Computation, vol. 139, no. 2-3, pp. 287-298, 2003.

[4] N. Koyuncu and C. Kadilar, "Family of estimators of population mean using two auxiliary variables in stratified random sampling," Communications in Statistics - Theory and Methods, vol. 38, no. 14, pp. 2398-2417, 2009.

[5] A. K. P. C. Swain, "On classes of modified ratio type and regression-cum-ratio type estimators in sample survey using two auxiliary variables," Statistics in Transition-New series, vol. 23, no. 3, pp. 473-494, 2012.

[6] J. Lu and Z. Yan, "A class of ratio estimators of a finite population mean using two auxiliary variables," PLos One, vol. 9, no. 2, pp. 1-6, Article ID e89538, 2014.

[7] J. Lu, Z. Yan, and X. Peng, "A new exponential ratio-type estimator with linear combination of two auxiliary variables," Plos One, vol. 9, no. 12, 10 pages, Article ID e116124, 2014 b.

[8] A. Sanaullah, M. Noor-ul-Amin, and M. Hanif, "Generalized exponential type ratio cum product and product cum product estimator for population mean in the presence of nonresponse stratified two-phase random sampling," Pakistan Journal of Statistics, vol. 31, no. 1, pp. 71-94, 2015.

[9] J. Lu, "Efficient estimator of a finite population mean using two auxiliary variables and numerical application in agricultural, biomedical, and power engineering," Mathematical 
Problems in Engineering, vol. 2017, Article ID 8704734, 7 pages, 2017.

[10] S. Muneer, J. Shabbir, and A. Khalil, "Estimation of finite population mean in simple random sampling and stratified random sampling using two auxiliary variables," Communications in Statistics - Theory and Methods, vol. 46, no. 5, pp. 2181-2192, 2017.

[11] J. Shabbir and S. Gupta, "Estimation of finite population mean in simple and stratified random sampling using two auxiliary variables," Communications in Statistics - Theory and Methods, vol. 46, no. 20, pp. 10135-10148, 2017.

[12] T. J. Akingbade and F. C. Okafor, "A class of ratio-type estimator using two auxiliary variables for estimating the population mean with some known population parameters," Pakistan Journal of Statistics and Operation Research, vol. 15, no. 2, pp. 329-340, 2019.

[13] J. Shabbir and S. Masood, "An improved general class of estimators for finite population mean in simple random sampling," Communications in Statistics- Theory and Methods, 2020.

[14] J. Shabbir and S. Gupta, "A new improved difference-cumexponential ratio type estimator in systematic sampling two auxiliary variables," Journal of the National Science Foundation of Sri Lanka, vol. 48, no. 1, pp. 27-36, 2020 b.

[15] S. Bhushan, R. Gupta, S. Singh, and A. Kumar, "Some log-type classes of estimators using multiple auxiliary information," International Journal of Scientific Engineering and Research, vol. 8, no. 6, pp. 12-17, 2020.

[16] S. A. Lone, M. Subzar, and A. Sharma, "Enhanced estimators of population variance with the use of supplementary information in survey sampling," Mathematical Problems in Engineering, vol. 2021, Article ID 9931217, 8 pages, 2021.

[17] C. Kumari and R. K. Thaur, "An efficient log-type class of estimators using auxiliary information under double sampling," Journal of Statistics Applications \& Probability, vol. 10, no. 1, pp. 197-202, 2021.

[18] M. N. Murthy, Sampling Theory and Methods, Statistical Publishing Society, Calcutta, India, 2nd edition, 1967.

[19] R. Singh and N. S. Mangat, Elements of Survey Sampling, Kluwer Academic Publishers, Amsterdam, Netherlands, 1996. 